\title{
Method of multifractal analysis of seismic noise
}

V. N. Sychev ${ }^{1}$

B. K. Dolgopolov ${ }^{2}$

S. A. Imashev ${ }^{1}$
${ }^{1}$ Research Station of Russian Academy of Sciences in Bishkek City, Bishkek, Kyrgyzstan

${ }^{2}$ Kyrgyz-Russian Slavic University named after B.N. Yeltsin, Bishkek, Kyrgyzstan

DFA (Detrended Fluctuation Analysis - Analysis of fluctuations after removing of scale-dependent trends) method of multifractal analysis of signal is described. Improved method of trend removal (detrending) based on discrete wavelet transform is proposed and algorithm of singularity spectrum boundaries estimation is modified. Analysis of dynamics of signal multifractal characteristics by an example of seismic noise is carried out.

\section{Keywords}

Multifractal analysis, Fractal dimension, Hurst exponent, Wavelet analysis, Detrending, Time series, Singularity spectrum

\section{References}

For citation: Sychev V.N., Dolgopolov B.K., Imashev S.A. Method of multifractal analysis of seismic noise. Geosystems of Transition Zones, 2017, vol. 1, N 2, p. 62-68. doi:10.30730/25418912.2017.1.2.062-068 